\title{
Comparison of prostate MRI-3D transrectal ultrasound fusion biopsy for first-time and repeat biopsy patients with previous atypical small acinar proliferation
}

\author{
Derek W. Cool, MD, PhD;' Cesare Romagnoli, MD; Jonathan I. Izawa, MD;' Joseph Chin, MD;, Lori Gardi, \\ David Tessier, PhD; ${ }^{3}$ Ashley Mercado, MD; Jonathan Mandel, MD; Aaron D. Ward, PhD, ${ }^{4}$ Aaron Fenster, PhD ${ }^{3}$
}

Department of Medical Imaging; ${ }^{2}$ Department of Surgery, Division of Urology; ${ }^{3}$ Robarts Research Institute; ${ }^{4}$ Department of Biophysics; University of Western Ontario, London, ON, Canada

Cite as: Can Urol Assoc J 2016;10(9-10):342-8. htrp://dx.doi.org/10.5489/cuaj.3831

\section{Abstract}

Introduction: This study evaluates the clinical benefit of magnetic resonance-transrectal ultrasound (MR-TRUS) fusion biopsy over systematic biopsy between first-time and repeat prostate biopsy patients with prior atypical small acinar proliferation (ASAP).

Materials: 100 patients were enrolled in a single-centre prospective cohort study: 50 for first biopsy, 50 for repeat biopsy with prior ASAP. Multiparameteric magnetic resonance imaging (MP-MRI) and standard 12-core ultrasound biopsy (Std-Bx) were performed on all patients. Targeted biopsy using MRI-TRUS fusion (Fn-Bx) was performed $f$ suspicious lesions were identified on the prebiopsy MP-MRI. Classification of clinically significant disease was assessed independently for the Std-Bx vs. Fn-Bx cores to compare the two approaches.

Results: Adenocarcinoma was detected in 49/100 patients (26 first biopsy, 23 ASAP biopsy), with 25 having significant disease (17 first, 8 ASAP). Fn-Bx demonstrated significantly higher per-core cancer detection rates, cancer involvement, and Gleason scores for first-time and ASAP patients. However, Fn-Bx was significantly more likely to detect significant cancer missed on Std-Bx for ASAP patients than first-time biopsy patients. The addition of Fn-Bx to Std-Bx for ASAP patients had a $166.7 \%$ relative risk reduction for missing Gleason $\geq 3+4$ disease (number needed to image with MP-MRI=10 patients) compared to $6.3 \%$ for first biopsy (number to image $=50$ patients). Negative predictive value of MP-MRI for negative biopsy was $79 \%$ for first-time and $100 \%$ for ASAP patients, with median followup of $32.1 \pm 15.5$ months.

Conclusions: MR-TRUS Fn-Bx has a greater clinical impact for repeat biopsy patients with prior ASAP than biopsy-naïve patients by detecting more significant cancers that are missed on Std-Bx.

\section{Introduction}

Multiparametric prostate magnetic resonance imaging (MP-MRI) is more sensitive than transrectal ultrasound (TRUS) for early-stage prostate adenocarcinoma (CaP), and may facilitate targeted biopsy to enhance detection..$^{1-12}$ Targeted biopsy of MP-MRI lesions can be performed directly using $M R I^{8-10}$ or under ultrasound guidance using cognitive targeting or MRI-TRUS fusion software. ${ }^{6,13}$ Needle insertion can be transrectal or transperineal under ultrasound guidance. MRI-targeted, TRUS-guided biopsy is likely best performed through fusion of MRI to 3D TRUS (MRI-TRUS fusion) rather than attempting to cognitively infer the MRI lesion location on standard non-fusion TRUS. . $^{14-16}$ While MRI-guided and MRI-TRUS fusion approaches have merit, MRI-TRUS fusion biopsy (Fn-Bx) allows for real-time needle visualization and can be performed in a clinical setting using a standard 2D TRUS probe incorporated into a 3D system, ${ }^{13}$ rather than in an MRI suite with specialized MRI-compatible hardware. ${ }^{8}$

$\mathrm{Fn}-\mathrm{Bx}$ has demonstrated higher per-core $\mathrm{CaP}$ detection rates, higher Gleason grades, and higher percentage of tumour involvement within targeted cores as compared to systematic biopsy..$^{2-7,12,17}$ However, clinical impact analysis on focused populations is needed to assess which patient populations (e.g., first biopsy vs. repeat biopsy) benefit most from MRI to optimize the use of MP-MRI and Fn-Bx resources within the diagnostic pathway. Patients with prior biopsy findings of atypical small acinar proliferation (ASAP) are a unique subset of the repeat biopsy population in whom repeat biopsy cancer detection rates $(40 \%)$ are similar to those seen in a low-risk, biopsy-naïve population (40\%). ${ }^{18,19}$ The benefit of Fn-Bx in the ASAP repeat biopsy population may not be the same as repeat patients with prior negative biopsy and requires independent evaluation. The similar cancer detection rates between first-time biopsy and ASAP repeat biopsy patients allows for direct comparison.

Our prospective cohort study compared repeat prostate biopsy patients with ASAP histology with first-time biopsy patients to evaluate the impact of prostate $\mathrm{Fn}-\mathrm{Bx}$ on $\mathrm{CaP}$ detection for low-risk populations. Since repeat biopsy patients more frequently harbour disease in prostatic regions 
less sampled by standard 12-core ultrasound biopsy (Std-Bx) protocol (e.g., anterior or medial posterior), we hypothesize that Fn-Bx is more likely for ASAP patients than for biopsynaïve patients to detect significant $\mathrm{CaP}$ misclassified or negative on Std-Bx.

\section{Methods}

\section{Study design}

A prospective double cohort study was approved by the institutional research ethics board. One hundred patients were prospectively enrolled from September 2011 to March 2014. The 50 patients within the first cohort were scheduled for first prostate biopsy based on prostate-specific antigen (PSA) levels/digital rectal exan (DRE) abnormalities alone. The second cohort of 50 patients had at least one prior biopsy that demonstrated ASAP and ongoing clinical concern for malignancy. Inclusion criteria for both cohorts were PSA 2-20 ng/L and no prior prostate MRI. Exclusion criteria were known CaP diagnosis and contraindication to MP-MRI or TRUS biopsy.

\section{MP-MRI}

Pre-biopsy MP-MRI was performed at 3T using endorectal and surface coils (GE Healthcare, Waukesha, WI) to acquire T2-weighted (T2W), diffusion-weighted, and dynamic contrast enhanced images; an additional axial T2W sequence was performed without endorectal coil and used for fusion to 3D TRUS (Table 1). MP-MRI was interpreted by a uroradiologist with six years and over 200 cases of MP-MRI

\begin{tabular}{|c|c|}
\hline \multicolumn{2}{|c|}{$\begin{array}{l}\text { Table 1. Multiparametric MRI sequence parameters } \\
\text { (sequences were performed with endorectal coil and an } \\
\text { additional T2W scan was performed without endorectal } \\
\text { coil using identical parameters) }\end{array}$} \\
\hline \multirow{5}{*}{ Axial T2W fast-spin echo } & TE/TR: 160/4343 \\
\hline & Slice thickness: $2.2 \mathrm{~mm}$ \\
\hline & Gap: $0 \mathrm{~mm}$ \\
\hline & FOV: $14.0 \mathrm{~cm}$ \\
\hline & Matrix: $320 \times 192,4$ averages \\
\hline \multirow{5}{*}{$\begin{array}{l}\text { Diffusion-weighted echo- } \\
\text { planar imaging (DWI) }\end{array}$} & b value: 100,800 \\
\hline & Slice thickness: $3.6 \mathrm{~mm}$ \\
\hline & Gap: 0 mm \\
\hline & FOV: $14.0 \mathrm{~cm}$ \\
\hline & Matrix: 128 x 256 \\
\hline \multirow{8}{*}{$\begin{array}{l}\text { Dynamic contrast-enhanced } \\
\text { (DCE) spoiled }\end{array}$} & Contrast injection rate: $4 \mathrm{ml} / \mathrm{s}$ \\
\hline & Temporal resolution: $6 \mathrm{sec} / \mathrm{scan}$ \\
\hline & Flip angle: 120 \\
\hline & TE/TR: $1.5 / 3.1$ \\
\hline & Slice thickness: $3.0 \mathrm{~mm}$ \\
\hline & Gap: 0 mm \\
\hline & FOV: $14 \mathrm{~cm}$ \\
\hline & Matrix: 128 x 128 \\
\hline
\end{tabular}

experience, blinded to the locations of prior ASAP histology to minimize possible interpretation bias.

All MP-MRI abnormalities were prospectively stratified according to level of suspicion, with a low threshold set to initiate Fn-Bx. Since a standardized reporting system was not established at the commencement of this study, all prospectively identified MP-MRI lesions were retrospectively assigned a Prostate Imaging Reporting and Data System version 2 score (PIRADS v2) ${ }^{20}$ to permit comparison with other studies. PIRADS scoring was performed by a separate expert abdominal radiologist who was blinded to patient identification, clinical history, and histology. The original interpreting radiologist did not perform the retrospective PIRADS assignment, as he had performed nearly all of the Fn-Bx and reviewed all pathology results, which would have introduced substantial interpretation bias.

\section{D TRUS biopsy platform}

TRUS imaging and targeted biopsy were performed using a 3D TRUS biopsy system designed within our laboratory, with a needle-guidance error of $2.1 \pm 1.3 \mathrm{~mm}$ in phantom studies. ${ }^{21}$ The system attaches a standard $9 \mathrm{MHz}$, end-fire 2D TRUS probe (Philips, Seattle, WA, U.S.) to a mechanical linkage that provides a natural range-of-motion. The mechanical design is similar to the Artemis ${ }^{\circledR}$ system (Eigen, Grass Valley, CA, U.S.), with 3D TRUS images acquired in 3-7 seconds through rotation of the TRUS probe about its long axis. Following 3D TRUS acquisition, the system monitors the position of the TRUS probe within the pelvis and all fused suspicious MP-MRI lesions are displayed on the real-time TRUS images for needle guidance.

\section{MRI-3D TRUS fusion biopsy}

A pre-biopsy 3D TRUS volume was acquired at the time of the MRI to perform software-based MRI-3D TRUS fusion prior to the biopsy procedure. Pre-biopsy MRI-TRUS fusion allowed for meticulous cross-modality rigid registration of the T2W MRI with the 3D TRUS image using manually identified anatomical landmarks. A model-based, non-rigid registration of the prostate boundaries using thin-plate splines $^{22}$ was performed for cases of significant MRI vs. 3D TRUS surface deformation (Fig. 1).

Biopsy procedures were performed in the ultrasound department with local anesthesia. A 12-core, 18-gauge Std-Bx was performed free-hand, with an additional core collected bilaterally from the anterior transitional zones (12 +2 cores) for ASAP patients only, which reflects standard clinical practice for all repeat biopsy patients at our institution. For those patients with suspicious lesions on MP-MRI, Fn-Bx was performed prior to Std-Bx, with 1-3 samples collected per lesion. MRI-TRUS fusion was performed using 

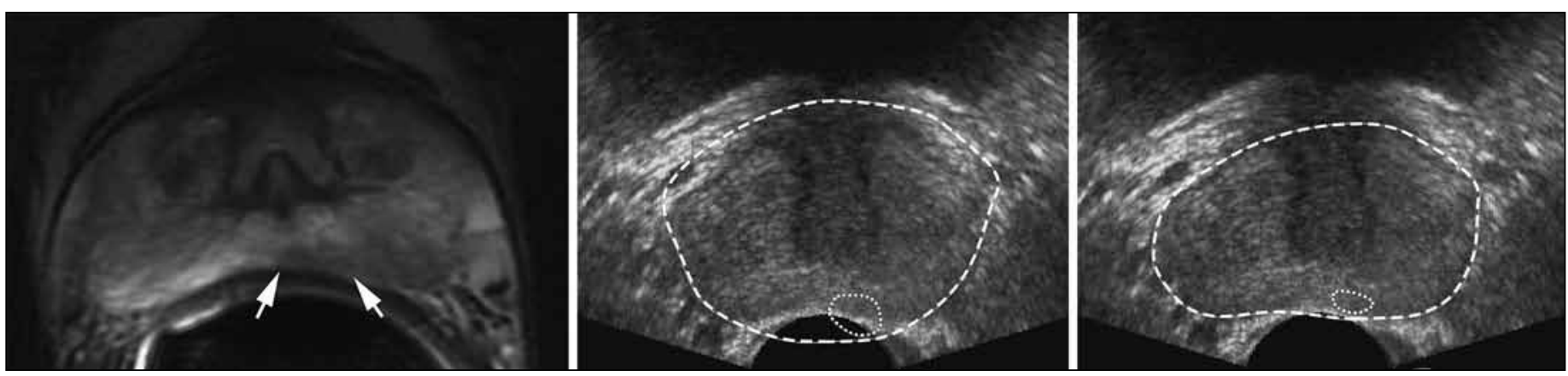

Fig. 1. Example of magnetic resonance imaging-transrectal ultrasound fusion of (MRI-TRUS) (A) a lesion identified on multiparametric -MRI in medial left mid-gland (arrow). Single slice of the 3D-TRUS image after only rigid registration of the MRI shows (B) poor correspondence of the MRI prostate boundary (dashed outline) and suspicious lesion (dotted outline) with the TRUS prostate gland. (C) Improved correspondence is achieved following thin-plate spline non-rigid alignment of the MRI and TRUS prostate surfaces. MRI-TRUS fusion biopsy demonstrated Gleason score $3+4,>90 \%$ core involvement.

3D TRUS-3D TRUS image-based registration algorithm ${ }^{23,24}$ to align the pre-biopsy 3D TRUS volume to the intra-biopsy 3D TRUS image. All MRI lesions were then mapped through the fusion pipeline and displayed on the intra-biopsy 3D TRUS platform for needle guidance.

\section{Evaluation of clinical significance}

The Fn-Bx core pathology results were interpreted independently from the Std-Bx cores to allow for comparison of the clinical impact using the Std-Bx as the internal control. The Gleason grade and percentage of tumour involvement was characterized for each positive core. Results were stratified as: no malignancy (ASAP included), clinically insignificant CaP, or clinically significant CaP. This study classified a core with Gleason score $\geq 3+4$ as clinically significant; however, clinical impact assessment used multiple clinical significance thresholds. ${ }^{25}$

\section{Data analysis}

Data were analyzed using the START criteria, ${ }^{26}$ including proportion of significant positive cores, mean number of cores taken per significant CaP diagnosis, and cross-tabulation of the detection of significant and insignificant $\mathrm{CaP}$ between Std-Bx and Fn-Bx. The MR lesion locations within the prostate were defined retrospectively based on the consensus 16-station reporting scheme. ${ }^{27}$ SPSS version 22.0 (IBM, Armonk, NY, U.S.) was used for statistical analysis with $\alpha=0.05$. Cancer detection percentages and the rates of significant $\mathrm{CaP}$ detection for Std-Bx and Fn-Bx were compared using Fischer's exact test. Mann-Whitney $U$ test evaluated the percentage of malignant core involvement. Detection of significant CaP from Fn-Bx + Std-Bx vs. Std-Bx alone was used for absolute (ARR) and relative risk reduction (RRR) calculations to determine the number needed to image (NNI) with MP-MRI to identify one clinically significant cancer that would have been missed on Std-Bx alone.

\section{Results}

\section{Cancer detection}

Patient demographics are summarized in Table $2 ; 76 \%$ of first-time biopsy patients (38/50) and $80 \%$ of ASAP patients (40/50) had MRI abnormalities, while 60\% (30/50) and 40\% $(20 / 50)$ of patients, respectively, had PIRADS $\geq 3$. In total, 125 suspicious lesions (first-time 69, ASAP 56) were identified (1.6 \pm 0.6 lesions/patient), of which 61 (first-time 30, ASAP 31 ) were prospectively classified as moderately or highly suspicious, with 68 classified as PIRADS $\geq 3$ (firsttime 45, ASAP 23) on retrospective assignment. Median time between MRI and biopsy was eight days (interquartile range [IQR] 8-15 days).

CaP was detected in 49 men (49\%; first-time 26, ASAP 23 ), with 25 patients ( $25 \%$; first-time 17 , ASAP 8 ) having Gleason $\geq 3+4$ (Table 3). Per-core CaP detection rates were significantly higher $(\mathrm{p}<0.001)$ for $\mathrm{Fn}-\mathrm{Bx}$ compared to StdBx (Fig. 2) and the relationship held for first-time and ASAP patients (Fig. 3). The per-core detection rate of significant CaP from Std-Bx was significantly lower $(p<0.001)$ for ASAP

\begin{tabular}{|c|c|c|}
\hline $\begin{array}{l}\text { Study patient } \\
\text { demographics }\end{array}$ & $\begin{array}{l}\text { First biopsy } \\
\qquad(n=50)\end{array}$ & $\begin{array}{l}\text { Repeat biopsy } \\
\text { with prior ASAP } \\
(n=50)\end{array}$ \\
\hline Age & $59.4 \pm 7.7$ years & $61.9 \pm 6.5$ years \\
\hline PSA & $6.0 \pm 3.5 \mathrm{ng} / \mathrm{ml}$ & $7.9 \pm 3.9 \mathrm{ng} / \mathrm{ml}$ \\
\hline Prostate volume & $38 \pm 18 \mathrm{~g}$ & $56 \pm 27 \mathrm{~g}$ \\
\hline $\begin{array}{l}\text { Number of prior } \\
\text { biopsies }\end{array}$ & None & $\begin{array}{c}1.7 \pm 0.9 \text { (median: } \\
1 \text {, range: } 1-5)\end{array}$ \\
\hline $\begin{array}{l}\text { Patients with MR } \\
\text { lesions }\end{array}$ & $\begin{array}{c}38 \text { (76\% of } \\
\text { patients) }\end{array}$ & $\begin{array}{l}40 \text { ( } 80 \% \text { of } \\
\text { patients) }\end{array}$ \\
\hline MR lesions per patient & $1.8 \pm 0.7$ lesions & $1.4 \pm 0.5$ lesions \\
\hline Biopsies per lesion & $\begin{array}{c}1.9 \pm 0.4 \text { cores } / \\
\text { lesion }\end{array}$ & $\begin{array}{c}1.9 \pm 0.6 \text { cores } / \\
\text { lesion }\end{array}$ \\
\hline Mean cores per patient & $14.9 \pm 1.8$ cores & $15.0 \pm 1.7$ cores \\
\hline
\end{tabular}


MRI-TRUS fusion biopsy for first-time and repeat biopsy

patients $(1.1 \% ; 7 / 618$ cores) compared to first-time biopsy patients $(8.1 \%$; 49/608 cores). However, Fn-Bx cores did not significantly differ $(p=0.39)$ in rates of significant CaP detection, with $15.2 \%$ (15/99 cores) and $20.0 \%$ (27/134 cores) detection for ASAP and first-time cohorts, respectively. When CaP was detected in both Std-Bx and Fn-Bx, the $\mathrm{Fn}$-Bx cores contained a significantly higher $\mathrm{CaP}$ volume $(p<0.05)$ for ASAP and first-time patients (Table 4).

\section{Clinically significant CaP detection}

The distribution of clinically significant CaP from Fn-Bx differed between the populations, with most of the ASAP patient disease located in the anterior prostate, in regions often undersampled during Std-Bx (Fig. 4). A significantly higher proportion $(p<0.01)$ of the clinically significant $\mathrm{CaP}$ (Gleason $\geq 3+4$ in this study) was detected only on Fn-Bx for ASAP patients compared to first-time biopsy patients. For first-time biopsy patients, only $1 / 17$ clinically significant cancers $(5.8 \%)$ was not accurately characterized on Std-Bx, compared to $5 / 8$ tumours $(62.5 \%)$ for ASAP patients (Table 5). The relatively higher proportions of significant CaP detected only on Fn-Bx for ASAP patients held for multiple definitions of significance (Table 6) and remained statistically significant for the more stringent significant $\mathrm{CaP}$ definition of Gls $\geq 4+3$ or core $>6 \mathrm{~mm}$ involved.

For ASAP patients, the addition of MP-MRI and Fn-Bx to Std-Bx resulted in RRR of missing Gleason $\geq 3+4$ disease of $167 \%$, an ARR of $10 \%$, and a NNI with MP-MRI of 10 patients to detect one significant CaP missed on Std-Bx. Fn-Bx for first-time biopsy patients had a RRR of $6 \%$, ARR of $2 \%$, and $\mathrm{NNI}$ of 50 patients. Fn-Bx had greater benefit for higher-grade definitions of clinical significance (Table 7).

\begin{tabular}{|c|c|c|c|}
\hline \multicolumn{4}{|c|}{ First biopsy patients } \\
\hline Highest Gleason & Equal in both & $\begin{array}{l}\text { Fn-Bx cores } \\
\text { highest }\end{array}$ & $\begin{array}{l}\text { Std-Bx cores } \\
\text { highest }\end{array}$ \\
\hline $3+3$ & 2 & 2 & 5 \\
\hline $3+4$ & 5 & 1 & 5 \\
\hline $4+3$ & 0 & 2 & 1 \\
\hline $4+4$ & 1 & 2 & 0 \\
\hline \multicolumn{4}{|c|}{ ASAP pepeat biopsy patients } \\
\hline Highest Gleason & Equal in both & $\begin{array}{l}\text { Fn-Bx cores } \\
\text { highest }\end{array}$ & $\begin{array}{l}\text { Std-Bx cores } \\
\text { highest }\end{array}$ \\
\hline $3+3$ & 1 & 3 & 11 \\
\hline $3+4$ & 2 & 3 & 0 \\
\hline $4+3$ & 0 & 2 & 0 \\
\hline $4+4$ & 0 & 1 & 0 \\
\hline
\end{tabular}

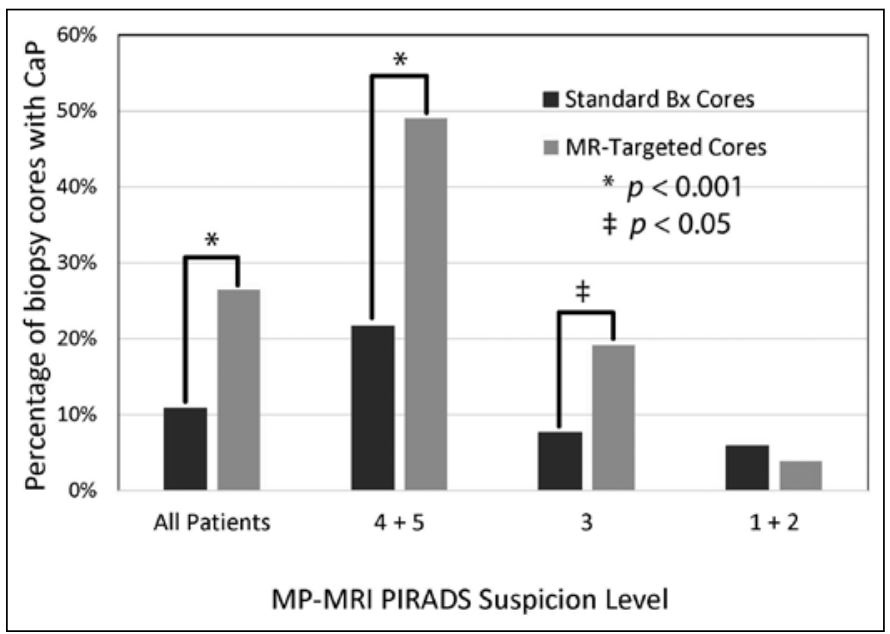

Fig. 2. Comparison of the percentage of biopsy cores positive for prostate adenocarcinoma ( $\mathrm{CaP}$ ) for both standard, systematic, and magnetic resonance-targeted biopsy approaches.

For ASAP patients, all clinically significant CaP was identified prospectively on MP-MRI and accurately characterized on Fn-Bx. If the Std-Bx was removed and only PIRADS $\geq 3$ lesions were targeted, then no significant $\mathrm{CaP}$ would have been missed, while $60 \%(30 / 50)$ of patients would have avoided biopsy and insignificant disease would have been found in three $(6 \%)$ instead of 15 patients (30\%).

For first-time biopsy, significant $\mathrm{CaP}$ was identified in six patients (12\%) on Std-Bx that was not diagnosed by Fn-Bx, with one deemed insignificant post-prostatectomy. Technical errors in MRI-3D TRUS fusion (intra-biopsy patient motion or inaccurate MRI-to-TRUS alignment) were possible causes for non-diagnostic targeted cores for three patients. The final two first-time biopsy patients had no prospectively identified suspicious findings on MP-MRI. For all patients with significant CaP found on Fn-Bx, at least insignificant CaP was detected on Std-Bx.

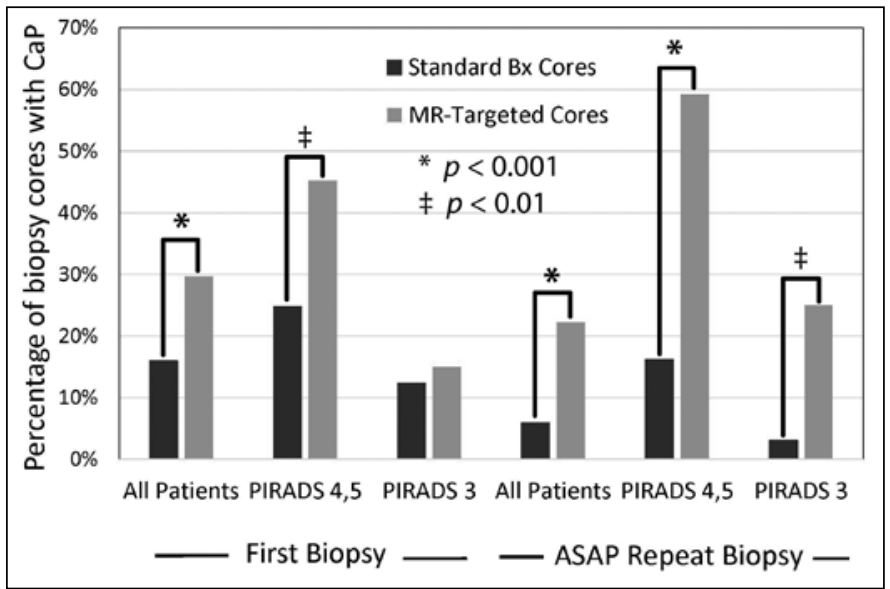

Fig. 3. Comparison of the percentage of biopsy cores positive for prostate adenocarcinoma ( $\mathrm{CaP}$ ) for both standard systematic and magnetic resonancetargeted biopsy approaches with first biopsy and atypical small acinar proliferation repeat biopsy patients separated. 
Cool et al.

\begin{tabular}{|c|c|c|c|}
\hline & $\begin{array}{l}\text { MRI-targeted } \\
\text { cores }\end{array}$ & $\begin{array}{c}\text { Standard } \\
\text { biopsy cores }\end{array}$ & p value \\
\hline All patients $(n=22)$ & $41.9 \pm 23.7 \%$ & $21.2 \pm 14.0 \%$ & $<0.01$ \\
\hline First biopsy ( $n=15)$ & $40.1 \pm 22.5 \%$ & $24.5 \pm 14.7 \%$ & $<0.01$ \\
\hline ASAP patients $(n=7)$ & $45.7 \pm 27.6 \%$ & $13.9 \pm 9.7 \%$ & $<0.01$ \\
\hline
\end{tabular}

\section{MP-MRI accuracy}

Median post-biopsy followup time was $32.1 \pm 15.5$ months (range 5-73 months). The negative predictive value (NPV) of a PIRADS 1 or 2 MP-MRI for negative biopsy or Gleason $3+3$ disease was $79 \%$ for first-time biopsy and $100 \%$ for ASAP patients and $73 \%$ and $100 \%$, respectively, throughout clinical followup. PIRADS $\geq 3$ was associated with a positive predictive value (PPV) of identifying significant $\mathrm{CaP}$ on biopsy or subsequent future biopsies of $50 \%$ for first-time patients and $40 \%$ for ASAP repeat patients. One in 15 patients with insignificant CaP $(6.7 \%)$ failed active surveillance and had T3a Gleason $3+4$ disease on prostatectomy. A total of seven active surveillance patients were biopsied during the followup period. Three of five patients who underwent repeat biopsy following negative or ASAP results on the study biopsy had low-volume Gleason $3+$ 4 disease. All four patients entered the study as first-time biopsy patients, three of whom had PIRADS=3 on MP-MRI.

\section{Discussion}

This study demonstrated that MRI-TRUS Fn-Bx was significantly more likely to upgrade benign or insignificant $\mathrm{CaP}$ on Std-Bx to significant CaP for ASAP patients compared to first-time biopsy patients. The addition of Fn-Bx to Std-Bx provided greater risk reduction of missing Gleason $\geq 3+4$ disease for ASAP patients (RRR 167\%, ARR 10\%, NNI 10) than for first-time biopsy patients (RRR 6.3\%, ARR 2.0\%, NNI 50). For biopsy-naïve patients, the addition of Fn-Bx reclassified one patient to significant disease from insignificant $\mathrm{CaP}$ on Std-Bx, despite the Fn-Bx cores having higher Gleason grades and tumour volumes than the Std-Bx cores. The superior Gleason grading and tumour volumes of $\mathrm{Fn}-\mathrm{Bx}$ observed for the first-time patients were similar to most prior studies. ${ }^{1,3-6,12,17}$ The benefit of Fn-Bx to first-time patients for detecting significant disease missed on Std-Bx only became evident at higher thresholds of cancer significance (e.g., Gleason $\geq 4+3$ and/or $\geq 6 \mathrm{~mm}$ core involved).

For biopsy-naïve patients, the limited reclassification to significant $\mathrm{CaP}$ for Fn-Bx is likely secondary to the posterior peripheral zone location of most of the significant cancers, where Std-Bx was able to sufficiently (though not optimally) sample the tumour. This suggests that for con-

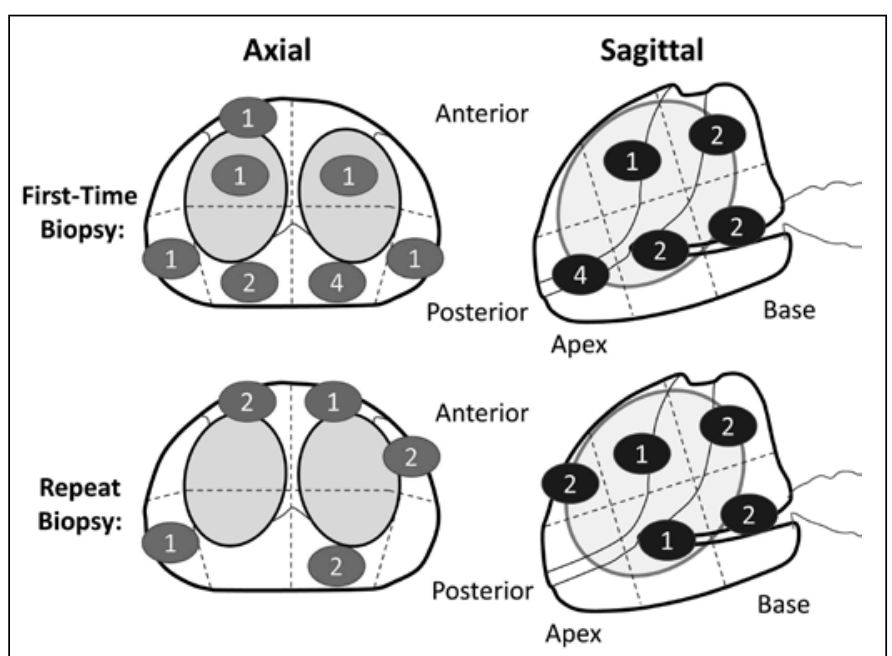

Fig. 4. Diagram illustrating the number clinically significant cancers detected in each prostate section as detected by magnetic resonance imaging-transrectal ultrasound fusion for first biopsy patients (top row) and atypical small acinar proliferation repeat biopsy patients (bottom row).

servative significance definitions (e.g., Epstein criteria or Gleason $\geq 3+4)$, if a Std-Bx will be performed regardless of MP-MRI findings, then MP-MRI for Fn-Bx is unlikely to substantially increase the detection of significant disease in first-time biopsy patients. While our sample size is not sufficient to detect small differences in significant $\mathrm{CaP}$ detection between the two techniques, the lack of such a difference for first-time biopsy patients is supported in recent randomized control studies comparing MRI-targeted and non-targeted TRUS biopsy. ${ }^{28,29}$ Conversely, recent non-randomized prospective studies by Mozer ${ }^{30}$ and Peltier ${ }^{31}$ did demonstrate a significant increase in significant $\mathrm{CaP}$ detection from $\mathrm{Fn}-\mathrm{Bx}$ for first-time biopsy patients using a low significance thresh-

Table 5. Cross-tabulation of the biopsy findings from MRI-TRUS fusion biopsy cores (rows) and from standard systematic biopsy cores (columns) for first-time and ASAP biopsy patients ${ }^{28}$

\begin{tabular}{lccc}
\hline & \multicolumn{3}{c}{ Standard biopsy core findings } \\
\hline First biopsy (n=50) & $\begin{array}{c}\text { No } \\
\text { cancer }\end{array}$ & $\begin{array}{c}\text { Insignificant } \\
\text { CaP }\end{array}$ & $\begin{array}{c}\text { Significant } \\
\text { CaP }\end{array}$ \\
\hline No MRI target & 10 & 1 & 1 \\
MRI-targeted biopsy findings & & 4 & 3 \\
No cancer & 14 & 2 & 2 \\
Insignificant CaP & 2 & 1 & 10 \\
Significant CaP & 0 & & \\
ASAP repeat biopsy (n=50) & & 4 & 0 \\
No MRI target & 6 & & \\
MRI-targeted biopsy findings & & 7 & 0 \\
No cancer & 21 & 1 & 0 \\
Insignificant CaP & 3 & 4 & 3 \\
Significant CaP & 1 & & \\
\hline ASAP:atypals
\end{tabular}

ASAP: atypical small acinar proliferation; CaP: prostate adenocarcinoma; MRI: magnetic resonance imaging; TRUS: transrectal ultrasound. 
MRI-TRUS fusion biopsy for first-time and repeat biopsy

\begin{tabular}{|c|c|c|c|}
\hline \multirow[b]{2}{*}{$\begin{array}{l}\text { Definition of clinical } \\
\text { significance }\end{array}$} & \multicolumn{3}{|c|}{$\begin{array}{l}\text { Percentage of significant CaP detected } \\
\text { (total number) }\end{array}$} \\
\hline & Fn-Bx alone & $\begin{array}{l}\text { Both Fn-Bx } \\
\& \text { Std-Bx }\end{array}$ & Std-Bx alone \\
\hline \multicolumn{4}{|c|}{ Gleason $\geq 3+4$ or core $\geq 50 \%$} \\
\hline First biopsy & $10.5 \%(2 / 19)$ & $57.9 \%(11 / 19)$ & $31.6 \%(6 / 19)$ \\
\hline ASAP biopsy & $40.0 \%(4 / 10)$ & $50.0 \%(5 / 10)$ & $10.0 \%(1 / 10)$ \\
\hline \multicolumn{4}{|l|}{ Gleason $\geq 3+4$ only } \\
\hline Firstbiopsy & $5.9 \%(1 / 17)$ & $58.8 \%(10 / 17)$ & $35.3 \%(6 / 17)$ \\
\hline ASAP biopsy & $62.5 \%(5 / 8)$ & $37.5 \%(3 / 8)$ & $0 \%(0 / 8)$ \\
\hline \multicolumn{4}{|c|}{ Gleason $\geq 4+3$ or core $\geq 6 \mathrm{~mm}$} \\
\hline First biopsy & $33.3 \%(5 / 15)$ & $46.7 \%(7 / 15)$ & $20 \%(3 / 15)$ \\
\hline ASAP biopsy & $87.5 \%(7 / 8)$ & $0 \%(0 / 8)$ & $12.5 \%(1 / 8)$ \\
\hline \multicolumn{4}{|l|}{ Gleason $\geq 4+3$ only } \\
\hline First biopsy & $60 \%(3 / 5)$ & $40 \%(2 / 5)$ & $0 \%(0 / 5)$ \\
\hline ASAP biopsy & $100 \%(3 / 3)$ & $0 \%(0 / 3)$ & $0 \%(0 / 3)$ \\
\hline
\end{tabular}

old (Gleason $\geq 3+3$ or $\geq 6 \mathrm{~mm}$ core involved). Siddiqui performed the largest prospective MRI-TRUS biopsy study to date ( $n=1003$ ) with subgroup analysis on 196 first-time patients, all of whom had positive MP-MRI findings. ${ }^{12}$ The study reported that the addition of Std-Bx to Fn-Bx did not significantly increase significant cancer detection $(3.6 \%$ of total cases); however, whether Fn-Bx increased significant $\mathrm{CaP}$ detection from Std-Bx alone was not discussed. Finally, a prospective internal control study by Pokorny ${ }^{32}$ also found increased detection of significant disease with MRI-guided biopsy, using a higher threshold for clinical significance (Gleason $\geq 4+3$ or $\geq 6 \mathrm{~mm}$ core involved), concordant with our study.

For ASAP patients, had the biopsy cores only been targeted at MP-MRI PIRADS $\geq 3$ lesions, then $40 \%$ of patients would have avoided biopsy and 12 insignificant cancers $(24 \%$ of patients) would not have been detected, without missing Gleason $\geq 3+4$ disease. Conversely, for first-time biopsy patients, removal of Std-Bx would have prevented detection of five $(10 \%)$ clinically insignificant $\mathrm{CaP}$, but would have missed five (10\%) Gleason $3+4$ malignancies (technical Fn-Bx errors the probable cause in three cases). Improved Fn-Bx technology, including intraprocedural motion compensation, ${ }^{33}$ are likely to minimize $\mathrm{Fn}-\mathrm{Bx}$ targeting errors and will be the focus of future studies.

To our knowledge, this is the first prospective study comparing the clinical impact of $\mathrm{Fn}-\mathrm{Bx}$ for repeat biopsy patients with prior ASAP histopathology to first-time biopsy patients. The study design enrolled patients without prior MP-MRI, supporting calculation of the NPV for patients with normal or benign MP-MRI findings - $79 \%$ for first-time biopsy and $100 \%$ for ASAP patients. Sonn et al prospectively compared

\begin{tabular}{|c|c|c|}
\hline $\begin{array}{l}\text { Definition of clinical } \\
\text { significance }\end{array}$ & $\begin{array}{l}\text { First-time biopsy } \\
\text { (RRR, ARR, NNI) }\end{array}$ & $\begin{array}{l}\text { Repeat biopsy with } \\
\text { ASAP (RRR, ARR, NNI) }\end{array}$ \\
\hline $\begin{array}{l}\text { Gleason } \geq 3+4 \text { or } \\
\text { core } \geq 50 \%\end{array}$ & $\begin{array}{l}11.8 \%, 4.0 \% \\
25 \text { patients }\end{array}$ & $\begin{array}{l}66.7 \%, 8.0 \% \\
12.5 \text { patients }\end{array}$ \\
\hline Gleason $\geq 3+4$ & $\begin{array}{l}6.3 \%, 2.0 \% \\
50 \text { patients }\end{array}$ & $\begin{array}{c}166.7 \%, 10.0 \%, \\
10 \text { patients }\end{array}$ \\
\hline $\begin{array}{l}\text { Gleason } \geq 4+3 \text { or } \\
\text { core } \geq 6 \mathrm{~mm}\end{array}$ & $\begin{array}{l}50 \%, 10 \%, \\
10 \text { patients }\end{array}$ & $\begin{array}{c}700 \%, 14.0 \%, \\
7 \text { patients }\end{array}$ \\
\hline Gleason $\geq 4+3$ & $\begin{array}{l}150 \%, 6 \% \text {, } \\
17 \text { patients }\end{array}$ & $\begin{array}{l}\text { None }^{*}, 6 \%, \\
17 \text { patients }\end{array}$ \\
\hline \multicolumn{3}{|c|}{$\begin{array}{l}{ }^{*} \text { No significant } \mathrm{CaP} \text { detected with Std-Bx alone. ARR: absolute risk reduction; ASAP: } \\
\text { atypical small acinar proliferation; CaP: prostate adenocarcinoma; Fn-Bx: magnetic } \\
\text { resonance imaging-transrectal ultrasound fusion biopsy; NNI: number needed to image; } \\
\text { RRR: relative risk reduction; Std-Bx: standard biopsy. }\end{array}$} \\
\hline
\end{tabular}

Fn-Bx with Std-Bx on 105 repeat biopsy patients (10 with ASAP) and demonstrated similar significant $\mathrm{CaP}$ detection rates $(21 \%$ of all patients vs. $16 \%$ in our study) and had five $(4.8 \%)$ significant cancers missed or mischaracterized with Fn-Bx. ${ }^{2}$ Our $46 \%$ total CaP yield for ASAP patients is consistent with the established $40 \%$ in the literature. ${ }^{18}$

Limitations of this study include lack of whole-gland histopathological assessment. Our study benefited from a long followup time (median $32 \pm 15$ months); however, indolent significant disease may still not have been declared and the calculated NPV may be falsely elevated. The prospective study size of 100 patients provides a good quantitative cohort comparison; however, larger multicentre studies are needed to confirm the results. The sample size was constrained by the limited number of available ASAP patients, despite the long study interval. Additionally, heterogeneity between the cohorts might have confounded the results, as the ASAP population had two additional cores sampled from the anterior prostate during the Std-Bx (total of 14 cores) and had slightly larger mean gland volumes (56 vs. $38 \mathrm{~mL}$ ), which has also been seen in larger studies with mixed populations. ${ }^{12}$ Extra biopsy cores for the ASAP patients would be expected to increase Std-Bx cancer detection and minimize the benefit of $\mathrm{Fn}-\mathrm{Bx}$ as compared to first-time patients, which was not evident in the results. Conversely, larger gland volumes decrease cancer detection rates for Std-Bx, which could have influenced the demonstrated superior benefit of Fn-Bx for ASAP patients. Finally, the prospective MP-MRI interpretation was not performed using a standardized system, such as PI-RADS, ${ }^{20}$ as no such system was established at study commencement; however, this was partly rectified by retrospective assignment.

\section{Conclusion}

MRI-TRUS fusion biopsy has a greater clinical impact on the detection of significant disease for repeat biopsy patients 
Cool et al.

with prior ASAP histology than for patients presenting for first biopsy.

Competing interests: Dr. Cool, Dr. Romagnoli, and Dr. Fenster have a license agreement with Eigen Medical Inc. Dr. Izawa has received speaker honoraria from Astellas, AstraZeneca, Janssen, and Sanofi. Dr. Chin was an advisor on the initial design and a principal investigator for US HIFU and Profound Medical Inc. The remaining authors report no competing personal or financial interests.

This paper has been peer-reviewed.

\section{References}

1. Delongchamps NB, Lefevre A, Bouazza N, et al. Detection of significant prostate cancer with magnetic resonance targeted biopsies — Should transrectal ultrasound-magnetic resonance imaging fusion-guided biopsies alone be a standard of care? J Urol 2015;193:1198-204. http://dx.doi.org/10.1016/i. juro.2014.11.002

2. Sonn $\mathrm{GA}$, Chang $\mathrm{E}$, Natarajan $\mathrm{S}$, et al. Value of targeted prostate biopsy using magnetic resonanceultrasound fusion in men with prior negative biopsy and elevated prostate-specific antigen. Eur Urol 2014;65:809-15. http://dx.doi.org/10.1016/.eururo.2013.03.025

3. Pinto PA, Chung PH, Rastinehad AR, et al. Magnetic resonance imaging/ultrasound fusion-guided prostate biopsy improves cancer detection following transrectal ultrasound biopsy and correlates with multiparametric magnetic resonance imaging. J Urol 2011;186:1281-5. http://dx.doi.org/10.1016/i. juro.2011.05.078

4. Sonn GA, Natarajan S, Margolis DJ, et al. Targeted biopsy in the detection of prostate cancer using an office-based magnetic resonance ultrasound fusion device. J Urol 2013;189:86-91. http://dx.doi. org/10.1016/i.juro.2012.08.095

5. Delongchamps NB, Peyromaure $M$, Schull $A$, et al. Prebiopsy magnetic resonance imaging and prostate cancer detection: Comparison of random and targeted biopsies. J Urol 2013;189:493-9. http://dx.doi. org/10.1016/i.juro.2012.08.195

6. Siddiqui MM, Rais-Bahrami S, Truong H, et al. Magnetic resonance imaging/ultrasound fusion biopsy significantly upgrades prostate cancer vs. systematic 12-core transrectal ultrasound biopsy. Eur Urol 2013;64:713-9. http://dx.doi.org/10.1016/i.eururo.2013.05.059

7. Kasivisvanathan V, Dufour R, Moore CM, et al. Transperineal magnetic resonance image targeted prostate biopsy vs. transperineal template prostate biopsy in the detection of clinically significant prostate cancer. J Urol 2013;189:860-6. http://dx.doi.org/10.1016/i.juro.2012.10.009

8. Anastasiadis AG, Lichy MP, Nagele U, et al. MRl-guided biopsy of the prostate increases diagnostic performance in men with elevated or increasing PSA levels after previous negative TRUS biopsies. Eur Urol 2006;50:738-48; discussion 48-9. http://dx.doi.org/10.1016/j.eururo.2006.03.007

9. Hoeks CM, Schouten MG, Bomers JG, et al. Three-Tesla magnetic resonance-guided prostate biopsy in men with increased prostate-specific antigen and repeated, negative, random, systematic, transrectal ultrasound biopsies: Detection of clinically significant prostate cancers. Eur Urol 2012;62:902-9. http:// dx.doi.org/10.1016/j.eururo.2012.01.047

10. Hambrock T, Somford DM, Hoeks C, et al. Magnetic resonance imaging-guided prostate biopsy in men with repeat negative biopsies and increased prostate-specific antigen. J Urol 2010; 183:520-7. http:// dx.doi.org/10.1016/i.juro.2009.10.022

11. Hadaschik BA, Kuru TH, Tulea C, et al. A novel stereotactic prostate biopsy system integrating preinterventional magnetic resonance imaging and live ultrasound fusion. J Urol 2011;186:2214-20. http:// dx.doi.org/10.1016/i.juro.2011.07.102

12. Siddiqui $M M$, Rais-Bahrami $S$, Turkbey $B$, et al. Comparison of MR/ultrasound fusion-guided biopsy with ultrasound-guided biopsy for the diagnosis of prostate cancer. JAMA 2015;313:390-7. http://dx.doi. org/10.1001/jama.2014.17942

13. Bax J, Cool D, Gardi L, et al. Mechanically assisted 3D ultrasound guided prostate biopsy system. Med Phys 2008;35:5397-410. http://dx.doi.org/10.1118/1.3002415

14. Cool DW, Zhang X, Romagnoli C, et al. Evaluation of MRI-TRUS fusion vs. cognitive registration accuracy for MRl-targeted, TRUS-guided prostate biopsy. AJR Am J Roentgenol 2015;204:83-91. http://dx.doi. org/10.2214/AJR.14.12681

15. Puech P, Rouviere 0 , Renard-Penna R, et al. Prostate cancer diagnosis: Multiparametric MR-targeted biopsy with cognitive and transrectal US-MR fusion guidance vs. systematic biopsy-prospective multicentre study. Radiology 2013;268:461-9. http://dx.doi.org/10.1148/radiol.13121501
16. Wysock IS, Rosenkrantz AB, Huang WC, et al. A prospective, blinded comparison of magnetic resonance (MR) imaging-ultrasound fusion and visual estimation in the performance of MR-targeted prostate biopsy: The PROFUS trial. Eur Urol 2014;66:343-51. http://dx.doi.org/10.1016/i.eururo.2013.10.048

17. Fiard $\mathrm{G}$, Hohn N, Descotes J-L, et al. Targeted MRI-guided prostate biopsies for the detection of prostate cancer: Initial clinical experience with real-ime 3-D transrectal ultrasound guidance and magnetic resonance/transrectal ultrasound image fusion. Urology 2013;81:1372-8. http://dx.doi.org/10.1016/i. urology.2013.02.022

18. Epstein Jl, Herawi M. Prostate needle biopsies containing prostatic intraepithelial neoplasia or atypical foci suspicious for carcinoma: Implications for patient care. J Urol 2006;175:820-34. http://dx.doi. org/10.1016/S0022-5347(05)00337-X

19. Ploussard G, Nicolaiew N, Marchand C, et al. Prospective evaluation of an extended 21-core biopsy scheme as initial prostate cancer diagnostic strategy. Eur Urol 2014;65:154-61. http://dx.doi.org/10.1016/i. eururo.2012.05.049

20. Weinreb JC, Barentsz J0, Choyke PL, et al. PI-RADS prostate imaging - Reporting and data system: 2015, Version 2. Eur Urol 2016;69:16-40. http://dx.doi.org/10.1016/i.eururo.2015.08.052

21. Bax J, Cool D, Gardi L, et al. Mechanically assisted 3D ultrasound guided prostate biopsy system. Med Phys 2008;35:5397-410. http://dx.doi.org/10.1118/1.3002415

22. Bookstein F. Principal warps: Thin-plate splines and the decomposition of deformations. IEEE Trans Pattern Anal Mach Intell 1989;11:567-85. htrp://dx.doi.org/10.1109/34.24792

23. Karnik WV, Fenster A, Bax J, et al. Assessment of image registration accuracy in three-dimensional transrectal ultrasound guided prostate biopsy. Med Phys 2010;37:802-13. http://dx.doi.org/10.1118/1.3298010

24. Karnik WV, Fenster A, Bax J, et al. Evaluation of intersession 3D-TRUS to 3D-TRUS image registration for repeat prostate biopsies. Med Phys 2011;38:1832-43. http://dx.doi.org/10.1118/1.3560883

25. Cooperberg MR, Carroll PR, Klotz L. Active surveillance for prostate cancer: Progress and promise. J Clin Oncol 2011;29:3669-76. http://dx.doi.org/10.1200/JC0.2011.34.9738

26. Moore CM, Kasivisvanathan V, Eggener $S$, et al. Standards of reporting for MRl-targeted biopsy studies (START) of the prostate: Recommendations from an International Working Group. Eur Urol 2013;64:54452. http://dx.doi.org/10.1016/i.eururo.2013.03.030

27. Dickinson L, Ahmed HU, Allen C, et al. Magnetic resonance imaging for the detection, localiszation, and characterization of prostate cancer: Recommendations from a European consensus meeting. Eur Urol 2011;59(4):477-94. http://dx.doi.org/10.1016/j.eururo.2010.12.009

28. Tontilia PP, Lantto J, Paakko E, et al. Prebiopsy multiparametric magnetic resonance imaging for prostate cancer diagnosis in biopsy-naive men with suspected prostate cancer based on elevated PSA values: Results from a randomized, prospective, blinded, controlled trial. Eur Urol 2016;69:419-25. http:// dx.doi.org/10.1016/i.eururo.2015.05.024

29. Baco E, Rud E, Eri LM, et al. A randomized, controlled trial to assess and compare the outcomes of two-core prostate biopsy guided by fused magnetic resonance and transrectal ultrasound images and traditional 12-core systematic biopsy. Eur Urol 2016;69:149-56. http://dx.doi.org/10.1016/i. eururo.2015.03.041

30. Mozer $\mathrm{P}$, Roupret $\mathrm{M}$, Le Cossec $\mathrm{C}$, et al. First round of targeted biopsies using magnetic resonance imaging/ ultrasonography fusion compared with conventional transrectal ultrasonography-guided biopsies for the diagnosis of localized prostate cancer. BJU Int 2015; 115:50-7. http://dx.doi.org/10.1111/biu.12690

31. Peltier $A$, Aoun F, Lemort $M$, et al. MRl-targeted biopsies vs. systematic transrectal ultrasound guided biopsies for the diagnosis of localized prostate cancer in biopsy naive men. Biomed Res Int 2015;2015:571708. http://dx.doi.org/10.1155/2015/571708

32. Pokorny MR, de Rooij M, Duncan E, et al. Prospective study of diagnostic accuracy comparing prostate cancer detection by transrectal ultrasound-guided biopsy vs. magnetic resonance (MR) imaging with subsequent MR-guided biopsy in men without previous prostate biopsies. Eur Urol 2014;66:22-9. http:// dx.doi.org/10.1016/j.eururo.2014.03.002

33. De Silva T, Fenster A, Cool DW, et al. 2D-3D rigid registration to compensate for prostate motion during 3D TRUS-guided biopsy. Med Phys 2013;40:022904. http://dx.doi.org/10.1118/1.4773873

Correspondence: Dr. Derek W. Cool, Department of Medical Imaging, University of Western Ontario, London, 0N, Canada; derek.coo@@gmail.com 